\title{
Incontinencia urinaria: causas y cuidados de enfermería. Una revisión bibliográfica
}

\author{
$M^{\text {a }}$ Nazaret Campillos-Cañete ${ }^{1}$, Rosa María González-Tamajón ${ }^{1}$, José Berlango-Jiménez ${ }^{1,2}$, \\ Rodolfo Crespo-Montero ${ }^{1,2,3}$ \\ ${ }^{1}$ Departamento de Enfermería. Facultad de Medicina y Enfermería. Universidad de Córdoba. Córdoba. España \\ ${ }^{2}$ Servicio de Nefrología. Hospital Universitario Reina Sofía de Córdoba. Córdoba. España \\ ${ }^{3}$ Instituto Maimónides de Investigación Biomédica de Córdoba. Córdoba. España
}

Como citar este artículo:

Campillos-Cañete MN, González-Tamajón RM, Berlango-Jiménez J, Crespo-Montero Rodolfo. Incontinencia urinaria: causas y cuidados de enfermería. Una revisión bibliográfica. Enferm Nefrol. 2021 Ene-Mar;24(1):25-37

\section{Resumen}

Introducción: La incontinencia urinaria ocurre con más frecuencia en el sexo femenino y conforme aumenta la edad, provocando una disminución de la calidad de vida.

Objetivo: Sintetizar la evidencia científica relacionada con las causas, tratamiento y cuidados de enfermería en la incontinencia urinaria.

Metodología: Se ha realizado una revisión bibliográfica sistematizada en las bases de datos Proquest, Pubmed, Google Académico y Scielo, utilizando como términos "incontinencia urinaria" (urinary incontinence), "factores de riesgo" (risk factor), "intervenciones de enfermería" (nursing intervention) y "procedimientos terapeúti$\cos ^{\prime \prime}$ (therapeutics), con una limitación de 5 años en la búsqueda. Se incluyeron estudios experimentales y de revisión.

Resultados: Se incluyeron 26 artículos: 5 estudios experimentales, 18 revisiones sistemáticas y 3 metaanálisis. La incontinencia urinaria está relacionada con la edad, sexo y factores de riesgo, dependiendo del tipo e intensidad de la incontinencia, y características propias del paciente. El abordaje es interdisciplinar, con técnicas menos invasivas al principio, entre las que destacan

\section{Correspondencia:}

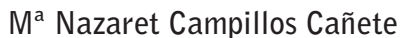

E-mail: nazaret23-4@hotmail.es la terapia conductual y los ejercicios del suelo pélvico; necesitándose cirugía en algunos casos, según las circunstancias individuales de los pacientes y/o la no existencia de mejoría con las técnicas menos invasivas.

Conclusiones: La incontinencia urinaria se encuentra estrechamente relacionada con la edad y el sexo; sin embargo, la existencia de factores de riesgo y algunas causas pueden contribuir a aumentar las probabilidades de su establecimiento. La labor enfermera resulta prometedora en el establecimiento de una detección y actuación precoz ante el problema, teniendo como base siempre para el tratamiento el menor grado de invasión.

PALABRAS CLAVE: incontinencia urinaria; factores de riesgo; síntomas; intervenciones de enfermería; terapéutica.

\section{Urinary incontinence: causes and nursing care. A bibliographic review}

\section{Abstract}

Introduction: Urinary incontinence is a disorder that occurs more in the female sex and with the increase of the age, producing as main consequence a decrease in the patient's quality of life. 
Objective: Synthesize the scientific evidence related to the causes, treatment and nursing care in urinary incontinence.

Methodology: A systematic bibliographic review was carried out in the ProQuest, PubMed, Google Academic and Scielo databases, using as terms "urinary incontinence", "risk factors", "nursing interventions" and "therapeutic procedures" with a 5-year search limitation. Experimental and review studies were included.

Results: 26 articles were included: 5 experimental studies, 18 systematic reviews and 3 meta-analyses. Urinary incontinence is affected by age, sex and risk factors depending on both the type and intensity of incontinence and the patient's own characteristics. The approach to this problem is an interdisciplinary one in which less invasive techniques are introduced, including behavioural therapy and pelvic floor exercises; to end up in surgery in case the patient's circumstances require it and/or there is no improvement with less invasive techniques.

Conclusions: Urinary incontinence is closely influenced by the variables age and sex; however, the existence of risk factors and some causes can contribute to increase the probability of their establishment. Nursing interventions are promising in establishing early detection and response to the problem, always based on the least degree of invasion.

KEYWORDS: urinary incontinence; risk factors; symptoms; nursing interventions; therapy.

\section{Introducción}

La incontinencia urinaria (IU) se considera un problema que acontece en nuestra sociedad y que afecta de una forma más considerable a la población femenina. De forma general, el 35\% de las mujeres experimenta algún tipo de IU a lo largo de cualquier etapa de su vida. Esta patología afecta negativamente a la calidad de vida de mujeres en etapa reproductiva, con un $20,4 \%$ de prevalencia en el climaterio; sin embargo, tiene un impacto más significativo cuando se llega a la edad media de 48 años, que suele coincidir con la instauración de la menopausia de la mayoría de las mujeres. Cuando avanzamos en edad cronológica, encontramos que la prevalencia en personas mayores de 70 años es mayor al $20 \%$, llegando a un $89,53 \%$ en aquellas mujeres que son mayores, dependientes y se encuentran en residencias ${ }^{1-5}$.

Las principales consecuencias que derivan de la IU son la depresión y el estrés; así como una disminución de la calidad de vida y un aumento de la mortalidad en aquellos rangos etarios más avanzados y vulnerables, como son las personas institucionalizadas ${ }^{2,4}$.

La probabilidad de que aparezca una IU aumenta con la edad, el sexo femenino, el asma y el uso de laxantes; influyendo también el incremento de parámetros antropométricos tales como la circunferencia de la cintura o el índice de conicidad, asociándose con aumento en la frecuencia e intensidad de las pérdidas ${ }^{1,6}$. Otros factores que pueden contribuir al aumento de la prevalencia de la IU, y por ello, se consideran de riesgo durante la época del embarazo y climaterio, son la obesidad, el estreñimiento, los problemas respiratorios, las cirugías como la episiotomía o el prolapso genital $\left.\right|^{4,7}$.

Enfermería es un colectivo que debe ser consciente de este tipo de peligros y supervisar los síntomas que más afectan a la vida del paciente. Entre ellos, debe conocer las contingencias que favorecen la aparición de la IU en las personas mayores que reciben atención hospitalaria. Así, se lleva a cabo una identificación de riesgos, lo cual impide que exista una subnotificación del problema. Estas actuaciones por parte de enfermería en el hospital, previenen que el paciente acabe con una IU en su hogar que provoque nuevos reingresos y pérdida de la calidad de vida ${ }^{5,8}$.

El tipo de IU que suele estar más presente en la población femenina es la mixta, siendo el resultado de la presencia de síntomas de IU de urgencia y de esfuerzo. El equipo sanitario, y enfermería en particular, debe comprender los tipos de IU para hacer una detección temprana que ayude al establecimiento de un tratamiento precoz 0 a la derivación a un especialista que pueda establecer un tratamiento médico; o en su defecto, quirúrgico, en caso de que se hayan probado ya las líneas de actuación primarias como serían: el entrenamiento de los músculos del suelo pélvico (SEPFM), el entrenamiento conductual o las modificaciones del estilo de vida. El personal enfermero debe conocer este tipo de actuaciones; ya que es el que se encuentra en estrecho contacto con la mayoría de los pacientes, conociéndolos más y estableciendo una continuidad de cuidados con éstos. Además, el establecimiento de una buena relación terapéutica ayuda a eliminar la vergüenza y la preocupación que derivan de hablar este tema que es considerado tabú en nuestra sociedad. Por lo tanto, enfermería actúa en el tratamiento conductual 
con la finalidad de aliviar los síntomas que más inciden en la rutina del paciente, y en caso de que este enfoque no lleve a una mejoría, se encargará de derivarlo al tipo de profesional más adecuado para que se establezca otro tipo de tratamiento $0^{5,8,9}$. Se demuestra también que una relación cercana con el paciente, un monitoreo continuo y el apoyo en los ejercicios del suelo pélvico son factores que mejoran la adherencia y los resultados, en caso de ser realizado por un equipo compuesto por diversas disciplinas que se enfocan en la resolución de la situación ${ }^{10}$.

Durante la hospitalización, el personal sanitario encargado de los cuidados debe dar visibilidad al tema y promover estrategias para evitar problemas como la iatrogenia durante el tiempo en que el paciente se encuentra hospitalizado. El equipo multidisciplinar debe promover actuaciones cognitivas, conductuales y sociales que ayuden a que determinados estados como el embarazo o circunstancias como la hospitalización, no se conviertan en factores de riesgo que acaben con el establecimiento de una IU9-11.

En resumen, la IU es un problema que puede establecerse en la población afectando a su calidad de vida o complicando las patologías principales que puedan existir de base. Se ha comprobado anteriormente que las líneas estratégicas que mayor eficacia conllevan son la realización de un reconocimiento y un abordaje precoz, que normalmente es establecido por el personal de enfermería que es el que mayor tiempo invierte por los cuidados que le presta. Este profesional también se encuentra en primera línea en el abordaje preventivo y terapéutico en el ámbito hospitalario. Por tanto, el objetivo principal de esta revisión fue sintetizar la evidencia científica relacionada con las causas, tratamiento y cuidados de enfermería en la IU, con los siguientes objetivos secundarios:

- Analizar las causas y los factores de riesgo que influyen en la IU.

- Conocer el manejo terapéutico y los cuidados de enfermería existentes para el tratamiento de los diferentes tipos de IU.

\section{Metodología}

\section{Diseño del estudio}

Se ha realizado una revisión bibliográfica sistematizada de la evidencia publicada, generando una síntesis narrativa de los artículos seleccionados. El procedimiento seguido ha sido: búsqueda bibliográfica, selección de artículos, evaluación de calidad, extracción e interpretación de los resultados.

\section{Estrategia de búsqueda}

La búsqueda bibliográfica se realizó en varias bases de datos con el fin de obtener la mayor producción científica disponible sobre el tema de estudio. Las bases de datos a las que se accedió fueron Pubmed/Medline, Scielo, ProQuest/Health and Medical Complete, la Biblioteca Cochrane y Google Académico. Se llevaron a cabo dos búsquedas bibliográficas, siendo el periodo de consulta de la primera búsqueda desde finales de últimos de diciembre de 2019 a finales de enero de 2020, y en la segunda, en el mes de febrero de 2020.

La búsqueda inicial se llevó a cabo en la base de datos de Google Académico y posteriormente, se accedió a Pubmed/Medline y Proquest/Health and Medical Complete, mientras que la segunda búsqueda se realizó en la base de datos Scielo.

El método de búsqueda fue diferente en función de las distintas bases de datos, empleando distintos métodos. Las palabras clave empleadas para la búsqueda en las diversas bases de datos fueron: incontinencia urinaria (urinary incontinence), factores de riesgo (risk factor), síntomas (symptoms), intervenciones de enfermería (nursing intervention) y procedimientos terapeúticos (therapeutics). Los términos MesH empleados en la búsqueda inicial a partir de los cuales se obtuvieron resultados fueron: ("Urinary incontinence" [MesH]) AND ("Therapeutic" [MesH]) AND ("risk factor" [MesH]), "nursing interventions", "theraphy". En la segunda búsqueda el término MesH utilizado fue: "Urinary Incontinence" [MesH].

\section{Criterios de inclusión / exclusión}

- Criterios de Inclusión: artículos que trataran como tema principal la IU, en los últimos 5 años desde el inicio de la búsqueda de documentos. Artículos de diseño experimental, revisiones sistemáticas y meta-análisis. Artículos en español e inglés.

- Criterios de exclusión: artículos sin texto completo disponible. Artículos referidos a la población infantil. Artículos cuyos resultados no fueran concluyentes.

\section{Selección de artículos}

Tras la realización de la lectura de los títulos, resúmenes y en ciertos casos, el artículo completo, se descartaron todos aquellos artículos que no cumplían con los criterios de inclusión/exclusión. 
Posteriormente, se requirió una segunda búsqueda, en la que se realizó el mismo proceso que en la primera, pero en la base de datos de Scielo; seleccionando los artículos de la misma forma escalonada, aplicando los criterios de inclusión/exclusión de forma gradual como en la primera búsqueda.

En la primera búsqueda se identificaron con las combinaciones MesH en las bases de datos un total de 688 artículos, de los cuales se preseleccionaron con los criterios de inclusión/exclusión 37. En la segunda búsqueda se identificaron un total de 209 artículos que tras aplicarle los criterios se redujeron a 58 artículos. Finalmente, se utilizaron para la revisión un total de 26 artículos, tal como puede observarse en la Figura 1.

\section{Síntesis de los resultados}

Se llevó a cabo la integración de las conclusiones obtenidas. Se empleó un estilo narrativo debido a la heterogeneidad de los estudios y a sus diferencias en cuanto a la evidencia científica relacionada con las causas, tratamiento y cuidados de enfermería en la IU.

\section{Resultados y discusión}

\section{- Características de los estudios}

Todos los artículos seleccionados fueron de diseño experimental, revisiones sistemáticas o metaanálisis: 5 estudios experimentales, 18 revisiones sistemáticas y 3 metaanálisis. En la Tabla 1 se muestran las principales características de los artículos seleccionados.

\section{- Descripción de las variables según los resul- tados}

\section{Prevalencia y complicaciones asociadas}

La bibliografía revisada muestra que la prevalencia de la IU es muy variable y depende estrechamente de las variables de edad y sexo. En el rango etario de mujeres que oscilan en una edad media de 47 años, los distintos tipos de IU se presentan con diferente frecuencia. En el caso de la IU de esfuerzo, se da en un $55 \%$ de los casos, seguida de la IU mixta con un $35,5 \%$, reseñándose ambas como las más relevantes de esta fase ${ }^{12}$.

Con respecto a la etapa etaria masculina comprendida entre los 45 y 75 años, los hombres suelen presentar una IU secundaria a una intervención quirúrgica, que en la mayoría de los casos resulta ser la prostatectomía. Según la técnica utilizada en la prostatectomía, además de la IU, puede ocurrir una dificultad añadida como es la disfunción erécti ${ }^{13,15}$.

Artículos incluidos para la redacción de los resultados $(n=26)$

En el caso de las mujeres con urgencia miccional, se ha observado que presentan complicaciones que se asocian a las pérdidas de orina, como son trastornos sexuales, que aparecen en un $86,6 \%$ de los casos. Entre ellos destacan: un 31\% presentan fugas durante el coito, $75 \%$ reflejan una altera-

Figura 1. Diagrama de flujo de la estrategia de búsqueda. ción en la excitación, $71 \%$ disminuye la lubricación y el $64 \%$ presentan trastornos en el orgasmo. Además, se ha descrito el $75 \%$ de mujeres que presentan urgencia miccional, refiere una frecuencia diurna elevada; así como más de dos eventos miccionales durante la noche, lo cual influye en su calidad de vida de forma reseñable ${ }^{12,14}$.

\section{De los artículos que se han seleccionado finalmente, se ha recopilado información relacionada con la IU (prevalen- cia, género y edad), causas o factores de riesgo, tratamien- to y cuidados de enfermería. \\ Análisis de artículos}


Tabla 1. Principales características de los artículos seleccionados.

\begin{tabular}{|c|c|c|c|}
\hline $\begin{array}{l}\text { Autores } \\
\text { Año }\end{array}$ & Tipo de estudio & $\mathbf{n}$ & Muestra Principales Resultados \\
\hline $\begin{array}{l}\text { Elaziz NAA, et } \text { al }^{14} \\
2016\end{array}$ & $\begin{array}{l}\text { Estudio } \\
\text { experimental }\end{array}$ & 190 & $\begin{array}{l}\text { Las modificaciones en el estilo de vida tienen un alto impacto en la } \\
\text { calidad de vida y en el bienestar psicológico en las mujeres ancianas con } \\
\text { incontinencia urinaria. }\end{array}$ \\
\hline $\begin{array}{l}\text { Hassan MA, et al }{ }^{45} \\
2015\end{array}$ & $\begin{array}{l}\text { Estudio cuasi- } \\
\text { experimental }\end{array}$ & 140 & $\begin{array}{l}\text { El programa de entrenamiento del suelo pélvico es efectivo en el } \\
\text { tratamiento de la IU y en la mejora de la calidad de vida de las mujeres. }\end{array}$ \\
\hline $\begin{array}{l}\text { FuY, et al }{ }^{39} \\
2019\end{array}$ & $\begin{array}{l}\text { Revisión sistemática } \\
\text { y síntesis narrativa }\end{array}$ & $16^{*}$ & $\begin{array}{l}\text { La IU puede ser tratada de forma útil con intervenciones basadas en } \\
\text { el comportamiento, ejercicios de la musculatura del suelo pélvico y } \\
\text { reentrenamiento de la vejiga. } \\
\text { En el futuro, las intervenciones conductuales pueden resultar como medidas } \\
\text { de autocontrol personalizadas. }\end{array}$ \\
\hline $\begin{array}{l}\text { Straczynska A, et al }{ }^{13} \\
2019\end{array}$ & Revisión sistemática & $8^{*}$ & $\begin{array}{l}\text { La IU tras una prostatectomía radical se ve tratada de forma efectiva por } \\
\text { el entrenamiento de los músculos del suelo pélvico. Mejoría en parámetros } \\
\text { físicos y calidad de vida. }\end{array}$ \\
\hline $\begin{array}{l}\text { Pannek J, et al }{ }^{19} \\
2017\end{array}$ & Revisión sistemática & 16 & $\begin{array}{l}\text { El cabestrillo mediouretral transobturador es una técnica de tratamiento } \\
\text { quirúrgico mínimamente invasiva con éxito en pacientes masculinos con } \\
\text { lesión de médula espinal. }\end{array}$ \\
\hline $\begin{array}{l}\text { Haglind } \mathrm{E} \text {, et } \mathrm{al}^{15} \\
2015\end{array}$ & $\begin{array}{l}\text { Ensayo no } \\
\text { aleatorizado } \\
\text { prospectivo }\end{array}$ & 2625 & $\begin{array}{l}\text { La prostatectomía laparoscópica asistida por robot preservó la función } \\
\text { eréctil frente a la técnica de prostatectomía radical retropúbica en el } \\
\text { tratamiento de los pacientes con cáncer de próstata. }\end{array}$ \\
\hline $\begin{array}{l}\text { Stewart F, et al }{ }^{31} \\
2017\end{array}$ & Revisión sistemática & $56^{*}$ & $\begin{array}{l}\text { Para la mejoría en la calidad de vida o cura de la IU de esfuerzo, parece } \\
\text { que la estimulación eléctrica se ha demostrado más efectiva que ningún } \\
\text { tratamiento activo o placebo. }\end{array}$ \\
\hline $\begin{array}{l}\text { Thomas LH, et } \mathrm{al}^{20} \\
2019\end{array}$ & Revisión sistemática & $20^{*}$ & $\begin{array}{l}\text { En el accidente cerebrovascular los factores más influyentes para la IU son: } \\
\text { estado de continencia, tiempo transcurrido y recurrencia del accidente. } \\
\text { La estimulación nerviosa eléctrica transcutánea y las terapias conductuales } \\
\text { puede reducir el número de episodios de incontinencia. }\end{array}$ \\
\hline $\begin{array}{l}\text { Imamura } M \text {, et al }{ }^{40} \\
2015\end{array}$ & Revisión sistemática & $11^{*}$ & $\begin{array}{l}\text { La pérdida de peso en mujeres con obesidad mórbida y moderada, estima } \\
\text { una mejoría de la incontinencia urinaria hasta los } 6 \text { y } 18 \text { meses después. } \\
\text { Por lo tanto, es factible considerarlo como tratamiento inicial antes que } \\
\text { otros tratamientos. }\end{array}$ \\
\hline $\begin{array}{l}\text { Ford } A A \text {, et al }{ }^{29} \\
2017\end{array}$ & Revisión sistemática & $81^{*}$ & $\begin{array}{l}\text { Las operaciones de sling uretral medio tienen son seguras y efectivas para } \\
\text { el tratamiento de la IU de esfuerzo en mujeres a corto y medio, y sobre todo } \\
\text { a largo plazo. Tiene además un impacto positivo en la calidad de vida. } \\
\text { Sin embargo, la técnica del transobsturador ha resultado más rentable que } \\
\text { la retropúbica y se derivan de ella menos efectos adversos. La técnica de las } \\
\text { cintas retropúbicas ha resultado más efectiva de abajo hacia arriba. }\end{array}$ \\
\hline $\begin{array}{l}\text { Woodley SJ, et al }{ }^{42} \\
2017\end{array}$ & Revisión sistemática & $38^{*}$ & $\begin{array}{l}\text { Las mujeres prenatales que son continentes o se desconoce su estado de } \\
\text { continencia al inicio del embarazo y realizan un programa estructurado } \\
\text { de entrenamiento de los músculos del suelo pélvico, pueden reducir la } \\
\text { incontinencia urinaria al final del embarazo y después del parto. Este tipo } \\
\text { de ejercicios son más efectivos en mujeres de mediana edad. }\end{array}$ \\
\hline $\begin{array}{l}\text { Dumoulin C, et } I^{41} \\
2018\end{array}$ & Revisión sistemática & $31^{*}$ & $\begin{array}{c}\text { El entrenamiento de los músculos del suelo pélvico reduce la cantidad de } \\
\text { fugas en las mujeres con IU de esfuerzo, cura o mejora los síntomas de } \\
\text { todos los tipos de IU, sobre todo la de esfuerzo. } \\
\text { Se puede proponer estos ejercicios como tratamiento conservador de } \\
\text { primera línea en mujeres con IU. }\end{array}$ \\
\hline
\end{tabular}




\begin{tabular}{|c|c|c|c|}
\hline $\begin{array}{l}\text { Autores } \\
\text { Año }\end{array}$ & Tipo de estudio & $\mathbf{n}$ & Muestra Principales Resultados \\
\hline $\begin{array}{l}\text { Nambiar A, et al }{ }^{27} \\
2017\end{array}$ & Revisión sistemática & $31^{*}$ & $\begin{array}{c}\text { El "sling de incisión única" (TVT-Secur) mostró tasas de incontinencia } \\
\text { mayores en mujeres que las cintas transobturadoras de "adentro hacia } \\
\text { afuera" y las retropúbicas (cinta vaginal sin tensión); aunque con } \\
\text { menor duración del tiempo de intervención quirúrgica y menor dolor } \\
\text { postoperatorio. } \\
\text { Los eventos adversos del "sling de incisión única" fueron más graves y los } \\
\text { principales son: mayor exposición a la malla vaginal, erosión vesical/uretral } \\
\text { y pérdida de sangre operativa. } \\
\text { Al final, TVT-Secur fue retirado del uso clínico. }\end{array}$ \\
\hline $\begin{array}{l}\text { Lima SVC, et al } \\
2018\end{array}$ & $\begin{array}{l}\text { Ensayo clinico no } \\
\text { aleatorizado }\end{array}$ & 15 & $\begin{array}{l}\text { El esfínter artificial resulta un método eficiente y seguro para el control de } \\
\text { la IU después de la prostatectomía radical. }\end{array}$ \\
\hline $\begin{array}{l}\text { Sun X, Yang et } \mathrm{al}^{28} \\
2015\end{array}$ & $\begin{array}{l}\text { Revisión sistemática } \\
\text { y meta-análisis }\end{array}$ & $16^{*}$ & $\begin{array}{l}\text { Las cirugías tanto de sling transobturador como retropúbico, resultaron } \\
\text { igual de efectivas para el tratamiento de la IU de esfuerzo; sin embargo, el } \\
\text { sling transobturador se asoció con una disminución del riesgo de padecer } \\
\text { complicaciones postoperatorias. }\end{array}$ \\
\hline $\begin{array}{l}\text { Moroni } R \text {, et a }{ }^{32} \\
2016\end{array}$ & $\begin{array}{l}\text { Revisión sistemática } \\
\text { y meta-análisis }\end{array}$ & $37^{*}$ & $\begin{array}{l}\text { El tratamiento de primera línea para la IU de esfuerzo debe ser el } \\
\text { comportamiento del suelo pélvico y la terapia muscular. } \\
\text { Las técnicas de fisioterapia posteriores (biorretroalimentación, conos } \\
\text { vaginales, electroestimulación...) resultan solo efectivas si las mujeres son } \\
\text { capaces de contraer de forma voluntaria los músculos del suelo pélvico. }\end{array}$ \\
\hline
\end{tabular}

\section{Espitia De LaHoz FJ,} et $\mathrm{al}^{12}$

2017

Ensayo clínico aleatorizado

45
Las pacientes sometidas a la rehabilitación del suelo pélvico son las que más mejoran su respuesta sexual; sin embargo, las mujeres que se han sometido al láser han presentado mayores tasas de resolución de la IU.

Para el tratamiento de la IU se aconseja al principio un tratamiento conservador o de cirugía menos invasiva que provoque el menor número de ingresos hospitalarios y haya una mayor reinserción laboral, social y sexual.

\begin{tabular}{|c|c|c|c|}
\hline $\begin{array}{l}\text { Matsuoka } P \text {, et al } \\
2019\end{array}$ & $\begin{array}{c}\text { Revisión sistemática } \\
\text { y meta-análisis }\end{array}$ & 9* & $\begin{array}{c}\text { La reparación del prolapso al mismo tiempo de la realización de cualquier } \\
\text { procedimiento profiláctico antiincontinencia, resultó efectivo para reducir } \\
\text { la incidencia de IU de esfuerzo en el postoperatorio de mujeres con } \\
\text { corrección quirúrgica del prolapso anterior o apical con IU asintomática. } \\
\text { La técnica de colposuspensión de Burch no mostró disminución de la IU de } \\
\text { esfuerzo después de la operación. }\end{array}$ \\
\hline $\begin{array}{l}\text { Zaidan } P \text {, et al }{ }^{23} \\
2016\end{array}$ & Revisión sistemática & $5^{*}$ & $\begin{array}{c}\text { Los ejercicios de los músculos del suelo pélvico resultan efectivos para el } \\
\text { tratamiento de la IU tras prostatectomía radical, mucho más cuanto antes } \\
\text { se empiecen en el postoperatorio. Su asociación a la electroestimulación no } \\
\text { afirma resultados adicionales. }\end{array}$ \\
\hline $\begin{array}{l}\text { Oliveira } \mathrm{M} \text {, et al }{ }^{43} \\
2017\end{array}$ & Revisión sistemática & $7^{*}$ & $\begin{array}{l}\text { El mejor tratamiento para la IU de esfuerzo en mujeres es la terapia de } \\
\text { fortalecimiento de los músculos del suelo pélvico junto a la palpación digital, } \\
\text { el monitoreo de la biorretroalimentación y los conos vaginales, incluyendo } \\
\text { parámetros como: entrenamiento durante } 12 \text { semanas de duración y } 10 \\
\text { repeticiones. }\end{array}$ \\
\hline
\end{tabular}

La forma para elegir qué técnica de cirugía se utilizará para la IU dependerá de varios factores entre ellos: cirugías abdominales previas o vaginales realizadas de forma concomitante, experiencia del cirujano, cirugías previas del paciente,

Oliveira LM, et $\mathrm{al}^{30}$ 2018
Meta-análisis
$48 *$ eventos adversos y disponibilidad de materiales.
Los sling pubovaginales dieron mejores resultados objetivos y subjetivos que las cirugías de colposuspension de Burch; sin embargo, mostraron mayor retención que provocó un regreso a la sala de operaciones.

Los sling retropúbicos obtienen mejores resultados objetivos y subjetivos a los sling transobturadores; no obstante, también presentan mayor número de efectos adversos. 


\begin{tabular}{|c|c|c|c|}
\hline $\begin{array}{l}\text { Autores } \\
\text { Año }\end{array}$ & Tipo de estudio & $\mathbf{n}$ & Muestra Principales Resultados \\
\hline $\begin{array}{l}\text { Carvalho MRD, et al }{ }^{38} \\
2018\end{array}$ & Revisión sistemática & 9* & $\begin{array}{c}\text { Para tratar la incontinencia en hombres post-prostatectomía resulta } \\
\text { efectivo el entrenamiento de los músculos del suelo pélvico con y sin } \\
\text { biorretroalimentación y la estimulación eléctrica. En aquellos hombres con } \\
\text { dificultad para localizar los músculos del suelo pélvico pueden comenzar con } \\
\text { sesiones de electroestimulación biofeedback. } \\
\text { Una mayor integración de la enfermera en el cuidado de la continencia } \\
\text { en estos pacientes mejorará la atención mediante el establecimiento de } \\
\text { ejercicios de los músculos del suelo pélvico y terapia conductual. }\end{array}$ \\
\hline $\begin{array}{l}\text { Saboia DM, et al }{ }^{44} \\
2018\end{array}$ & Revisión sistemática & $6^{*}$ & $\begin{array}{l}\text { Los ejercicios de entrenamiento de los músculos del suelo pélvico resultan } \\
\text { efectivos en el post-parto inmediato y tardío para la prevención de la } \\
\text { IU. Los programas que usan ejercicios con una contracción más fuerte, } \\
\text { de mayor tiempo de mantenimiento de ésta y con mayor número de } \\
\text { repeticiones resultan los más indicados. } \\
\text { La palpación vaginal digital resulta un método efectivo clínico en el } \\
\text { puerperio para medir la contracción de los músculos del suelo pélvico. }\end{array}$ \\
\hline $\begin{array}{l}\text { Matsuoka PK, et al }{ }^{33} \\
2016\end{array}$ & Revisión sistemática & $14 *$ & $\begin{array}{c}\text { La mayoría de los implantes que se inyectan a nivel de la uretra en las } \\
\text { mujeres adultas para el tratamiento de la IU de esfuerzo, parecen ser } \\
\text { seguros porque no mostraron efectos adversos muy graves, excepto la grasa } \\
\text { autóloga. } \\
\text { Las complicaciones más importantes de los implantes son a nivel urinario: } \\
\text { retención e infección del tracto urinario. }\end{array}$ \\
\hline $\begin{array}{l}\text { Longo PS, et al }{ }^{24} \\
2019\end{array}$ & Revisión sistemática & $11^{*}$ & $\begin{array}{l}\text { La histerectomía total resulta con menos impacto para la función urinaria } \\
\qquad \text { que la histerectomía subtotal. }\end{array}$ \\
\hline $\begin{array}{l}\text { Bretterbauer KM, et } \\
\text { al }^{25} \\
2016\end{array}$ & $\begin{array}{l}\text { Observacional } \\
\text { retrospectivo }\end{array}$ & 236 & $\begin{array}{c}\text { La mayoría de la muestra presenta una mejoría de la incontinencia tras } \\
\text { la intervención quirúrgica para la IU de esfuerzo, independientemente } \\
\text { de la cantidad de operaciones anteriores y el recuento preoperatorio de } \\
\text { compresas. La tasa de satisfacción permanece constante durante el tiempo } \\
\text { de estudio observado y hay ciertas características de los pacientes que } \\
\text { la aumenta: más jóvenes, alto nivel de actividad física, gran reducción de } \\
\text { compresas. }\end{array}$ \\
\hline
\end{tabular}

IU: Infecciones Urinarias. *: número de artículos revisados.

Otros autores, han comunicado cifras de prevalencia de IU, en el sexo femenino y edad avanzada del $43 \%$; resultando esta cifra en un $2,5 \%$ en el caso de la población femenina con edad adulta alrededor de los 37,7 años y de bajos ingresos. En éstas últimas, un $46 \%$ de ellas reveló que nunca habían oído hablar de la IU, el $66 \%$ no sabían que existía un tratamiento médico; además, alcanzaron cifras del $100 \%$ en el desconocimiento acerca de la existencia de un tratamiento fisioterapéutico ${ }^{16,17}$.

Asimismo, existen estudios que han investigado el período de la mujer gestante y puerperal, observando que la IU que con más frecuencia aparece en este período es la de esfuerzo, con un comienzo durante la gestación y una continuidad durante la etapa del postparto. En el supuesto de tratarse de mujeres jóvenes y de mediana edad, la IU de esfuerzo sigue siendo la más frecuente; sin embargo, cuando las mujeres llegan a ser personas mayores, la IU de urgencia y mixta es la que se presenta de forma más habitual ${ }^{18,26}$.

\section{Causas y/o factores de riesgo}

La presencia de numerosos factores predisponentes provoca una mayor probabilidad de establecimiento de una IU. Además, existen también factores que modifican la intensidad de la IU como puede ser la hipermovilidad uretral ${ }^{12}$.

De forma general, se consideran factores de riesgo en ambos sexos la obesidad y la presencia de factores irritantes (reír, toser, estornudar) en caso de tratarse del desarrollo de una IU de esfuerzo ${ }^{13,14}$.

De forma más específica, la presencia de comorbilidades (hipertensión, diabetes mellitus, hipercolesterolemia), lesión cervical por esfinterotomía y/o un accidente 
cerebrovascular influyen notablemente en el estado de continencia ${ }^{19-21}$.

La causa principal de la IU masculina suele ir asociada a intervención quirúrgica, resultando en un $89 \%$ de los casos tras una prostatectomía radical y en un $4 \%$ de los casos por otras intervenciones quirúrgicas. Tanto la prostatectomía laparoscópica asistida por robot como la prostatectomía radical retropúbica, producen tasas de incontinencia muy parecidas, $21 \%$ y $20 \%$ respectivamente. No obstante, la primera de las técnicas presenta una menor tasa de disfunción erécti $\left.\right|^{15,25}$

En el caso del sexo femenino, la presencia de laxitud vaginal, prolapso anterior mayor o igual a grado II 0 la histerectomía, resultan componentes que pueden conducir o aumentar el peligro de padecer una IU. En este último caso, se ha comunicado que la histerectomía total tiene un menor impacto que la subtotal en la función urinaria, lo cual induce a pensar que será un mejor tratamiento para aquellas enfermedades uterinas benignas ${ }^{12,22,24}$.

Otros investigadores apoyan con su trabajo, la obesidad como principal factor de riesgo estipulado para la IU de esfuerzo, añadiendo también otros como la diabetes mellitus, el factor genético, hormonas, medicamentos y el parto vaginal ${ }^{26}$.

En el caso de la IU postparto, estudios recientes evidencian que además de ser la IU durante el embarazo un factor de riesgo importante, añade otros tales como la multiparidad o la edad gestacional al nacimiento mayor o igual a 37 semanas $^{18}$.

Por lo que se refiere a la IU en las personas mayores, un estudio observacional en ancianos institucionalizados, muestra que el principal factor de riesgo en esta población es la incapacidad funcional, a la que pueden añadirse las comorbilidades, ya citadas anteriormente en nuestros resultados. Aunque tampoco debe olvidarse, factores como la restricción de movilidad y la dependencia funcional que influyen considerablemente en la continencia miccional ${ }^{17}$.

\section{Tratamiento}

El tratamiento para la IU más desarrollado y evidenciado es el tratamiento quirúrgico; debido a que el farmacológico, resultó tener una baja tasa de respuesta y se encontró poca información acerca de él en la búsqueda ${ }^{14}$.
Por un lado, el tratamiento con intervención quirúrgica de la IU de esfuerzo se puede realizar por distintas técnicas. EI TVT-Secur, es un dispositivo de Sling de incisión única, que, a pesar de tener una menor duración del tiempo de intervención, resultó tener malos resultados y elevadas complicaciones que hicieron que se retirara del uso clínico. En contraposición, el Sling mediouretral transobturador es un método mínimamente invasivo para el tratamiento de la IU de esfuerzo femenina que además puede ser utilizado en hombres que padezcan una lesión medular. Por consiguiente, esta técnica resulta más efectiva y con un perfil más alto de seguridad para el tratamiento de la IU a largo plazo. En comparación con la técnica de Sling mediouretral retropúbico, presenta un menor número de efectos adversos y complicaciones, entre los que destacan una disminución en la perforación de la vejiga, disfunción miccional y/o lesión vascular/medular. También, se ha destacado su eficiencia reflejada en parámetros tales como el escaso tiempo que dura la intervención y la breve estancia hospitalaria postoperatoria. No obstante, una de las principales dificultades de este procedimiento es el mayor riesgo de dolor postoperatorio en el muslo e ingle, que, aunque es de corta duración, es un aspecto a evaluar por parte del personal sanitario $0^{19,27-30}$.

La técnica de colposuspensión de Burch, resultó útil para la reducción de la incidencia en el postoperatorio de mujeres con prolapso anterior, siempre que se usara como técnica complementaria y sinérgica ante la cirugía principal que es la reparación del prolapso. En el tratamiento de la IU de esfuerzo, es un método con peores resultados objetivos y subjetivos que el Sling pubouretral. En cambio, éste último junto al Sling mediouretal retropúbico, resulta más retencionista provocando un mayor número de reintervenciones ${ }^{22,30}$.

Para el tratamiento de la IU no neurogénica y en hombres tras prostatectomía retropúbica radical, se muestra como una opción fiable, eficiente, satisfactoria y segura, el esfínter artificial; ya que se produce una mejoría tanto de la calidad de vida, como del mantenimiento del flujo urinario evitando pérdidas de orina y síntomas obstructivos derivados de la intervención. El nivel de satisfacción de esta técnica se ve incrementado con características del paciente tales como la juventud y el alto nivel de actividad física ${ }^{21-25}$.

Por otro lado, a nivel de tratamiento menos invasivo, encontramos la estimulación eléctrica (EE) que se ha mostrado más efectiva que la ausencia de tratamiento. Asimismo, la EE transcutánea unida a la terapia con- 
ductual reduce el número de episodios de incontinencia urinaria durante las 24 horas. Existen diferentes métodos, entre los que podemos encontrar la EE intravaginal, y la EE de superficie. Ambas refieren una mejoría de la calidad de vida y una disminución de las pérdidas de orina en el tratamiento de la IU de esfuerzo $20,31,32$.

La técnica de láser de $\mathrm{CO}_{2}$ fraccionado junto a la inyección uretral resultan beneficiosas en las tasas de resolución de la IU de esfuerzo en mujeres ${ }^{12,33}$.

Las evidencias aportadas por otros autores muestran que además de existir técnicas innovadoras, prometedoras y poco desarrolladas como el tratamiento de radiofrecuencia para la IU de esfuerzo; alertan también acerca de la influencia perjudicial de ciertos factores como la edad avanzada o el Índice Masa Corporal en técnicas ya expuestas en nuestros resultados como es el Sling mediouretral retropúbico y transobturador. Igualmente, apoyan la utilización de otras técnicas ya citadas, que son mínimamente invasivas como es el caso de la inyección parauretral. Resaltan su alta tasa de curación (60-70\%); así como su capacidad de ser seguras, efectivas y servir como tratamiento duradero (mínimo dos años) en otros tipos de IU como es la de urgencia $26,34-36$.

En el caso de los esfínteres artificiales, ciertos estudios cuestionan su efectividad en ciertos pacientes con características especiales, es decir, en los que se dé un grado de incontinencia grave y tasa de complicaciones grado III tras la realización de una prostatectomía radical ${ }^{37}$.

\section{Cuidados de enfermería: tratamiento conservador}

La integración del colectivo enfermero en el cuidado y la detección de la incontinencia resulta un aspecto clave; ya que mejora la atención prestada al paciente por parte del profesional sanitario. Al principio, en el abordaje de la IU, resulta recomendable comenzar con un tratamiento conservador, entre los que se encuentran los ejercicios de fortalecimiento de los músculos del suelo pélvico (SEPFM) y terapia conductual; para posteriormente, continuar con cirugías en caso de no experimentar mejoría. Las cirugías deben seguir una escala ascendente en el grado de invasión; puesto que el objetivo es disminuir en lo posible el número de ingresos hospitalarios junto a una mayor reinserción temprana tanto laboral como social, sin olvidar la sexual ${ }^{12,38}$.
La terapia conductual suele ser el tratamiento de primera línea y se ha referido que logra una mejora tanto en la calidad de vida como en el bienestar psicológico. Puede ser una estrategia utilizada como única actuación o también, puede ir asociada con otras intervenciones como ejercicios del suelo pélvico, reentrenamiento de vejiga o biofeedback. Asimismo, resulta útil en casos de IU cuyo origen es una causa distinta a la enfermedad neurogénica. Entre las principales actuaciones que se indican al paciente con esta terapia, encontramos una modulación de la ingesta de líquido tanto en cantidad como en sincronización de la ingesta, una disminución de la ingesta de la cafeína, una modificación saludable del cuidado del cuerpo; así como un tratamiento eficaz de la IU en situaciones sociales. De igual manera, en mujeres con obesidad mórbida que padezcan una IU, la principal acción que se debe indicar como terapia es la pérdida de peso; debido a que éste es el factor más relevante en este caso para mejorar de forma notable los síntomas y las fugas urinarias ${ }^{14,20,39,40}$.

En relación con el reentrenamiento de vejiga, es necesario que se adecue al estilo de vida de cada persona. De esta forma, habrá más probabilidad de que se muestre beneficioso logrando una reducción de los episodios por semana y una disminución en la cantidad de la pérdida de orina ${ }^{39}$.

Por lo que se refiere a los SEPFM, están indicados para cualquier tipo de IU, como tratamiento primario; pero pueden resultar más efectivos en aquellos casos de IU de esfuerzo y/o mujeres de edad avanzada. En el supuesto de las mujeres durante la gestación y el postparto, la sugerencia de utilización es como medida preventiva. En el caso de la IU post prostatectomía, se asocia que este tipo de ejercicios son más fructuosos si utilizamos previamente técnicas como la EE y/o los establecemos lo antes posible en el postoperatorio. En general, los SEPFM, suelen mostrar mayores tasas de mejora subjetiva, mejor respuesta sexual en el paciente, menores pérdidas de orina tanto en cantidad como en frecuencia; e incluso, mejor calidad de vida con disminución de la gravedad de la IU cuando se encuentran asociados a otras intervenciones como la $E E$, el biofeedback, el reentrenamiento de vejiga o conos vaginales ${ }^{12-14,23,32,38-45}$.

Hallazgos recientes llevados a cabo por autores que han analizado el problema de la IU, muestran y confirman que un tratamiento gradual comenzando por la parte más conservadora (SEPFM junto a dispositivos de asistencia como bioretroalimentación o conos vaginales) y progresando a tratamientos más invasivos, son actua- 
ciones que se encuentran respaldadas por numerosa evidencia. Además, aparte de apoyar nuestros resultados, añaden datos acerca del potencial negativo en la calidad de vida de un paciente que convive con este tipo de dificultad, como resultado del sentimiento de vergüenza, el miedo, la falta de confianza y autocontrol en las situaciones sociales. Esto pone en significación el papel del profesional sanitario debido a que es el que debe de realizar una evaluación clínica de la IU, incluyendo datos minuciosos sobre la historia centrada en el problema; lo cual ayudará a realizar una detección precoz que le permitirá escoger unas estrategias de afrontamiento adecuadas, resultando todo ello como un predictor de calidad de vida ${ }^{26,46}$.

\section{Limitaciones del estudio}

La búsqueda en sí misma ha resultado una limitación. A pesar de que existe una gran abundancia de evidencia científica, fue complicado el hecho de establecer unos criterios de inclusión y exclusión para conseguir un número de artículos "manejable" y con el mayor grado de evidencia para la realización del trabajo. Otras de las limitaciones ha sido encontrar información acerca de cuidados de enfermería. Este ámbito ha sido explorado a través de términos que hacen referencia al tratamiento conservador, corriendo el riesgo en algunos casos de solaparse con competencias de otros profesionales como es el caso de los fisioterapeutas. La escasez de investigación acerca del papel enfermero en este problema, hace que sus competencias no se encuentren claramente definidas.

\section{Consideraciones prácticas}

La IU resulta un problema, que en edades avanzadas llega a tomar tanta relevancia, que se considera un síndrome geriátrico. Se trata de un tema tabú que hace que la población sienta vergüenza, pudor, aprensión o miedo a compartirlo con el personal sanitario; y por consiguiente, hace que su subnotificación no permita una detección y abordaje precoz que impida que sus consecuencias se vuelvan más nefastas.

La IU incide de forma directa en una alteración de la esfera no solo física y psicológica; sino que también de la parte social que provoca que no tengan adecuadas herramientas para lidiar con los efectos propios de esta alteración. El personal sanitario debe poseer los conceptos básicos que le permitan acometer la dificultad en estadios primarios; y en caso de no ser de su competencia, tener la capacidad de derivar a otro profesional adecuado para que dé la respuesta que el paciente necesita.

La enfermería es un colectivo fundamental dentro del equipo interdisciplinar que debe abordar este problema; puesto que, en atención primaria, son la avanzadilla terapéutica que puede lograr conseguir una notificación y actuación precoz para que la persona goce de las medidas menos invasivas posible. La formación en terapia conductual y los mínimos conocimientos en SEPFM junto a un correcto establecimiento de una relación terapéutica, puede ayudar a luchar contra esta preocupación encubierta en nuestra sociedad.

A la vista de los resultados de esta revisión podemos afirmar que:

- La IU resulta un problema que depende de forma importante tanto de la edad como del sexo. Existen unos factores de riesgo y unas causas que influyen tanto de forma directa como indirecta aumentando la probabilidad de padecerla o en caso de estar establecida, aumentar su intensidad.

- El tratamiento va muy ligado al tipo de IU que se haya establecido y a las características específicas del paciente. De forma general, se recomienda un abordaje que comience con las técnicas menos invasivas y se irá aumentando el grado hasta llegar a la cirugía en caso de no paliar el problema.

- Enfermería resulta un colectivo prometedor que en caso de tener adquiridos los suficientes conocimientos y establecer una correcta relación terapéutica, lograría realizar una detección y abordaje precoz eliminando inconvenientes tales como la subnotificación del problema o la incorrecta actuación o derivación en el establecimiento del tratamiento.

Recepción: 16-08-20

Aceptación: 15-12-20

Publicación: 30-03-21 


\section{Bibliografía}

1. Sacomori C, Rodrigues-Vinter C, Flores-Sperandio F, Felden-Pereira É, Luiz-Cardoso F. Propuesta de puntos de corte para diferentes indicadores antropométricos en la predicción de la incontinencia urinaria en mujeres. Rev chil obstet ginecol. 2015; 80(3):229-35.

2. Borges $C L$, Fernandes $B K C$, Cavalcante MLSN, Barbosa RGB, Peixoto Junior AA, Menezes LCG de. Risk factors for urinary incontinence in institutionalized elderly. ESTIMA, Braz J Enterostomal Ther. 2019;e0619:10.

3. Rett MT, Wardini ÉB, Santana JM de, Mendonça $A C R$, Alves AT, Saleme CS. Female urinary incontinence: quality of life comparison on reproductive age and postmenopausal period. Fisioter mov. 2016;29(1):71-8.

4. Dellú MC, Schmitt $A C B$, Cardoso MRA, Pereira WMP, Pereira ECA, Vasconcelos É da SF, et al. Prevalence and factors associated with urinary incontinence in climacteric. Rev Assoc Med Bras. 2016;62(5):441-6.

5. Stewart E. Assessment and management of urinary incontinence in women. Nursing Standard. 2018; 33(2):75-81.

6. Junqueira JB, Santos VLC de G. Urinary incontinence in hospital patients: prevalence and associated factors. Rev Latino-Am Enfermagem. 2018;25:e2970.

7. Bekele $A$, Adefris $M$, Demeke S. Urinary incontinence among pregnant women, following antenatal care at University of Gondar Hospital, North West Ethiopia. BMC Pregnancy Childb. 2016;16(1):6.

8. Pair, LS, Somerall WE. Urinary incontinence: Pelvic floor muscle and behavioral training for women. The Nurs Pract. 2018;43(1):21-5.

9. Góes RP, Pedreira LC, David RAR, Silva CFT, Torres CAR, Amaral JB do. Hospital care and urinary incontinence in the elderly. Rev Bras Enferm. 2019;72(Suppl 2):S284-93.

10. Mendes A, Rodolpho JRC, Hoga LAK. Non-pharmacological and non-surgical treatments for female urinary incontinence: an integrative review. Appl Nurs Res. 2016;31:146-53.
11. Bezerra K de C, Rocha SR, Oriá MOB, Vasconcelos CTM, Sabóia DM, Kc B, et al. Interventions for the prevention of urinary incontinence during prenatal care: An Integrative review. Online Brazilian Journal of Nursing. 2016;15(1):1-10.

12. Espitia De La Hoz FJ, Orozco Gallego H. Evaluación de la mejoría de la sexualidad en mujeres intervenidas por incontinencia urinaria. Rev Peru Ginecol Obstet. 2017;63(4):537-46.

13. Straczynska A, Weber-Rajek M, Strojek K, Piekorz Z, Styczynska H, Goch A, et al. The Impact of Pelvic Floor Muscle Training On Urinary Incontinence In Men After Radical Prostatectomy (RP) - A Systematic Review. CIA. 2019;14:1997-2005.

14. Elaziz NAA, Mahmoud S. Impact of Lifestyle Modification Program on Quality of Life, and Psychological Wellbeing among Elderly Women with Urinary Incontinence. Public Policy Admin Research. 2016;13:64-76.

15. Haglind E, Carlsson S, Stranne J, Wallerstedt A, Wilderäng $U$, Thorsteinsdottir $T$, et al. Urinary Incontinence and Erectile Dysfunction After Robotic Versus Open Radical Prostatectomy: A Prospective, Controlled, Nonrandomised Trial. Eur Urol. 2015;68(2):216-25.

16. Zago AC, Fambrini MAS, Silva EPG, Vitta AD, Conti MHSD, Marini G. Prevalence and knowledge of urinary incontinence and possibilities of treatment among low-income working women. Fisioter mov. 2017;30(Suppl 1):S151-9.

17. Roig JJ, De Souza DLB, Lima KC. Urinary incontinence in institutionalized elderly: prevalence and impact on quality of life. Fisioter mov. 2015;28(3):583-96.

18. Leroy $L$ da $S$, Lúcio $A$, Lopes MHB de M. Risk factors for postpartum urinary incontinence. Rev esc enferm USP. 2016;50(2):200-7.

19. Pannek J, Wöllner J. Treatment of stress urinary incontinence in men with spinal cord injury: minimally invasive $=$ minimally effective? Spinal Cord 2017; 55(8):739-42.

20. Thomas LH, Coupe J, Cross LD, Tan AL, Watkins $\mathrm{CL}$. Interventions for treating urinary incontinence after stroke in adults. Cochrane DB Syst Rev. 2019 Feb $1 ; 2(2)$ :CD004462. 
21. Lima SVC, De Barros EGC, Vilar $F$ de 0, Pinto FCM, Barros TDP, Truzzi JC, et al. Artificial sphincter "BR - SL - AS 904" in the treatment of urinary incontinence after radical prostatectomy: efficacy, practicality and safety in a prospective and multicenter study. Int Braz J Urol. 2018;44(6):121523.

22. Matsuoka $P$, Castro R, Baracat E, Haddad J. Occult Urinary Incontinence Treatment: Systematic Review and Meta-analysis-Brazilian Guidelines. Rev Bras Ginecol Obstet. 2019;41(02):116-23.

23. Zaidan P, Silva EB da. Pelvic floor muscle exercises with or without electric stimulation and post-prostectomy urinary incontinence: a systematic review. Fisioter mov. 2016;29(3):635-49.

24. Longo PS, Borbily LV, Glina FPA. Urinary incontinence following subtotal and total hysterectomy: a systematic review. Einstein (São Paulo). 2019 May 2;17(2):eRW432017.

25. Bretterbauer KM, Huber ER, Remzi M, Huebner W. Telephone - delivered quality of life after 365 male stress urinary incontinence (SUI) operations. Int Braz J Urol. 2016;42(5):986-92.

26. Sanders K. Treatment of Stress Urinary Incontinence in Women: A Medical and Surgical Review. Urol Nurs. 2019;39(1):29-35.

27. Nambiar A, Cody JD, Jeffery ST, Aluko P. Single-incision sling operations for urinary incontinence in women. Cochrane DB Syst Rev. 2017 Jul 26;7(7):CD008709.

28. Sun X, Yang Q, Sun F, Shi Q. Comparison between the retropubic and transobturator approaches in the treatment of female stress urinary incontinence: a systematic review and meta-analysis of effectiveness and complications. Int Braz J Urol. 2015; 41(2):220-9.

29. Ford AA, Rogerson $L$, Cody JD, Aluko P, Ogah JA. Mid-urethral sling operations for stress urinary incontinence in women. Cochrane DB Syst Rev. 2017 Jul $31 ; 7(7): C D 006375$.

30. Oliveira L, Dias M, Martins S, Haddad J, Girão M, Castro R. Surgical Treatment for Stress Urinary Incontinence in Women: A Systematic Review and Meta-analysis. Rev Bras Ginecol Obstet. 2018; 40(08):477-90.
31. Stewart F, Berghmans B, Bø K, Glazener CMA. Electrical stimulation with non implanted devices for stress urinary incontinence in women. Cochrane DB Syst Rev. 2017 Dec 22;12(12):CD012390.

32. Moroni R, Magnani P, Haddad J, Castro R, Brito L. Conservative Treatment of Stress Urinary Incontinence: A Systematic Review with Meta-analysis of Randomized Controlled Trials. Rev Bras Ginecol Obstet. 2016;38(02):97-111.

33. Matsuoka P, Locali R, Pacetta A, Baracat E, Haddad J. The efficacy and safety of urethral injection therapy for urinary incontinence in women: a systematic review. Clinics. 2016;70(2):94-100.

34. Lordelo $P$, Boas AV, Sodré $D$, Lemos A, Tozetto $S$, Brasil C. New concept for treating female stress urinary incontinence with radiofrequency. Int Braz J Urol. 2017;43(5):896-902.

35. Laterza RM, Halpern K, Ulrich D, Graf A, Tamussino K, Umek W, et al. Influence of age, BMI and parity on the success rate of midurethral slings for stress urinary incontinence. PLoS ONE. 2018; 13(8):e0201167.

36. De Vries AM, van Breda HMK, Fernandes JG, Venema PL, Heesakkers JPFA. Para-Urethral Injections with Urolastic for Treatment of Female Stress Urinary Incontinence: Subjective Improvement and Safety. Urol Int. 2017;99(1):91-7.

37. Dos Santos-Junior ACS, Oliveira-Rodrigues L, Castelo-Azevedo D, Almeida-Carvalho LM, Ribeiro-Fernandes M, Oliveira-Sapori Avelar $S$, et al. Artificial urinary sphincter for urinary incontinence after radical prostatectomy: a historical cohort from 2004 to 2015. Int Braz J Urol. 2017; 43(1):150-4.

38. Carvalho MRD. Terapias alternativas para la recuperación temprana de la continencia urinaria posterior a la prostatectomía: una revisión sistemática. Enferm Global. 2018;15:1215-23.

39. Fu Y, Nelson EA, McGowan L. Multifaceted self-management interventions for older women with urinary incontinence: a systematic review and narrative synthesis. BMJ Open. 2019; 9:e028626.

40. Imamura $M$, Williams $K$, Wells $M$, McGrother $C$. Lifestyle interventions for the treatment of urinary incontinence in adults. Cochrane DB Syst Rev. 2015 Dec 2;(12):CD003505. 
41. Dumoulin C, Cacciari LP, Hay-Smith EJC. Pelvic floor muscle training versus no treatment, or inactive control treatments, for urinary incontinence in women. Cochrane DB Syst Rev. 2018 0ct 4; 10(10):CD005654.

42. Woodley SJ, Boyle R, Cody JD, Mørkved S, Hay-Smith EJC. Pelvic floor muscle training for prevention and treatment of urinary and faecal incontinence in antenatal and postnatal women. Cochrane DB Syst Rev. 2017 Dec 22; 12(12):CD007471.

43. Oliveira M, Ferreira M, Azevedo MJ, Firmino-Machado J, Santos PC. Pelvic floor muscle training protocol for stress urinary incontinence in women: A systematic review. Rev Assoc Med Bras. 2017; 63(7):642-50.
44. Saboia DM, Bezerra K de C, Vasconcelos Neto JA, Bezerra LRPS, Oriá MOB, Vasconcelos CTM. The effectiveness of post-partum interventions to prevent urinary incontinence: a systematic review. Rev Bras Enferm. 2018;71(Suppl 3):S1460-8.

45. Hassan Hussein MA, Mohamed HE-S, El-Nemer AMR. Pelvic floor muscle training program for Egyptian women with neglected urinary incontinence. JNEP. 2015;5(5):92-102.

46. Senra C, Pereira MG. Quality of life in women with urinary incontinence. Rev Assoc Med Bras. 2015; 61(2):178-83.

Este artículo se distribuye bajo una Licencia Creative Commons Atribución-NoComercial 4.0 Internacional. https://creativecommons.org/licenses/by-nc/4.0/ 